\title{
Técnicas de Comunicação em Projetos de Tecnologia da Informação: um estudo de caso no setor financeiro
}

\author{
IT Project Communication Techniques: a case study in the financial industry
}

\author{
Leandro Zocaratto Rezende ${ }^{1 \mathbf{i}}$ \\ Orcid: https://orcid.org/0000-0002-5543-9155
}

Edmir Parada Vasques Prado ${ }^{2 i i}$

Orcid: https://orcid.org/0000-0002-3505-6122

\begin{abstract}
Resumo
A comunicação desempenha papel importante no gerenciamento de projetos. Entretanto, muitos trabalhos acadêmicos têm destacado os problemas de comunicação como causa para insucesso de projetos. Neste contexto, esta pesquisa tem como objetivo analisar técnicas de comunicação em projetos de Tecnologia da Informação (TI) em uma instituição financeira brasileira, que é um dos setores da economia com maiores investimentos em projetos de TI. Para atingir esse objetivo, foi feita uma revisão sistemática da literatura sobre características e técnicas de comunicação. Esta pesquisa possui uma abordagem qualitativa e descritiva. Foram entrevistados 10 profissionais que atuam com projetos de TI, em uma instituição financeira de grande porte, em 2019. A pesquisa identificou as técnicas de comunicação mais usadas, entre as quais se destacam: correio eletrônico, mensagens de texto e reuniões em grupo. Diversas motivações para sua adoção foram identificadas: comunicação com stakeholder, questões de localização, grande quantidade de dados, e informalidade na comunicação.
\end{abstract}

Palavras-chave: técnicas de comunicação; projetos de tecnologia da informação. gestão de projetos;setor financeiro.

\begin{abstract}
Communication plays an important role in project management. Many academic surveys, however, have highlighted communication problems as a cause for project failure. Therefore, this study aims to analyze communication techniques in information technology (IT) projects in a Brazilian financial institution with one of the largest investments in IT projects. We then conducted a literature review on the characteristics and techniques of communication. This is a qualitative and descriptive study. We interviewed 10 professionals who work with IT projects in a large financial institution during the year of 2019. The survey identified the most widely used communication techniques, and amongst them, the following stand out: electronic mail, text messages, and group meetings. We also identified several motivations for the adoption of such techniques, including communication with stakeholders, location issues, large amounts of data, and informality in communication.
\end{abstract}

Keywords: communication techniques; information technology projects; project management; financial industry.

\footnotetext{
${ }^{1}$ Universidade de São Paulo, Brasil. E-mail: leandro.zocaratto@gmail.com

${ }^{2}$ Universidade de São Paulo, Brasil. E-mail: eprado@usp.br
} 


\section{INTRODUÇÃO}

As altas taxas de insucesso em projetos de tecnologia que envolvem desenvolvimento de software têm se tornado um problema generalizado, e muitos trabalhos acadêmicos têm atribuído as causas aos problemas relacionados à comunicação (LU; LIU; LIU, 2009). Para esses autores, a comunicação possui um papel importante não só entre os membros dos times de projetos, mas também entre eles e os clientes. De forma semelhante, Vasconcellos e Hemsley (2002) destacam que os problemas de comunicação é uma das principais fraquezas estruturais das empresas tradicionais, se tornando mais graves à medida que o nível hierárquico aumenta.

A comunicação desempenha um papel importante no gerenciamento de projetos e nas relações humanas. Gerentes de projeto passam cerca de $90 \%$ de seu tempo em atividades relacionadas ao gerenciamento das comunicações. Comunicar é um dos fatores críticos para o sucesso de um projeto (RUUSKA; VARTIAINEN, 2003). Dentro desse contexto, o estudo das técnicas de comunicação em projeto de tecnologia da informação (TI) se mostra relevante. Além disso, poucos trabalhos na literatura destacam os pontos fortes e fracos das técnicas de comunicação e sua contribuição para o sucesso em projetos. Nas bases de dados ACM Digital Library, IEEE Xplore Digital Library, Science Direct, Scopus e Web of Science, entre o período de 2010 a 2019, existem apenas onze trabalhos publicados sobre esse tema e nenhum deles aborda a realidade brasileira. Portanto, o presente trabalho pretende contribuir com essa lacuna de conhecimento analisando as técnicas de comunicação usadas em projeto de TI, considerando a realidade brasileira.

Para atender à questão de pesquisa "Qual a importância das principais técnicas de comunicação, utilizadas em projetos de TI, em uma instituição financeira brasileira de grande porte?" definiu-se o seguinte objetivo geral: analisar as técnicas de comunicação em projetos de TI em uma instituição financeira brasileira. O setor financeiro foi selecionado, pois segundo Meirelles (2015) os bancos representam um ramo da economia entre os mais informatizados e que relativamente mais gasta e investe em TI. Corrobora essa afirmação dados da Federação Brasileira de Bancos (FEBRABAN, 2020), que reportou investimentos de $\mathrm{R} \$ 8,6$ bilhões do setor em 2019.

Para atender a esse objetivo, foram definidos três objetivos específicos: (1) identificar, a partir de uma revisão sistemática da literatura, as principais técnicas de comunicação em projetos de TI; (2) confirmar as técnicas de comunicação, por meio de entrevistas, com profissionais com experiência em projetos de TI em uma instituição financeira brasileira; e (3) analisar a importância dessas técnicas em função da frequência de uso, das motivações para adotálas e de seus pontos fortes e fracos.

No que se refere à estrutura do artigo, primeiramente foi apresentado uma revisão da literatura sobre as características da comunicação e suas técnicas. Em seguida é descrito o método empregado na pesquisa, formando a base para a apresentação e discussão dos resultados. Por fim, são apresentadas as conclusões da pesquisa.

\section{REVISÃO DA LITERATURA}

Esta seção apresenta as características da comunicação e as técnicas de comunicação em projetos. Cabe destacar, inicialmente, o conceito de comunicação. A comunicação pode ser definida como um processo que envolve a transmissão e a recepção de mensagens entre uma fonte emissora e um destinatário receptor, no qual as informações são codificadas na fonte e decodificadas no 
destino, por meio de sistemas convencionados (CHAVES et al., 2008).

\subsection{Características da Comunicação em Projetos}

Inúmeros trabalhos na literatura caracterizaram a comunicação em projetos. Essas características foram feitas em função de diversos fatores:

Verbalização. Dinsmore e Cavalieri (2003) classificaram a comunicação, em termos de verbalização, em três categorias. A primeira categoria é a verbal. Nesse caso, a comunicação utiliza palavras e se subdivide em dois tipos: oral, por meio de palavras faladas, geralmente de forma rápida e clara, que permite manter a mensagem simples e sucinta, e possibilita a obtenção de feedback do receptor no momento da transmissão da mensagem; e escrita, que possui maior nível de detalhamento e é utilizada para explicar temas de maior complexidade ou com necessidade de formalização, possibilita melhor entendimento contínuo do receptor, deixando a mensagem à sua disposição para revisão e absorção. A segunda categoria é a não verbal. Nessa categoria a transmissão de mensagens é feita sem o uso de palavras, sinais vocais ou paralinguísticos, sendo de extrema utilidade na transmissão de signos. Exemplos dessa categoria são as emoções e sentimentos, tais como dor, cansaço, motivação, entre outras. Por último, a terceira categoria é a fisiológica ou paralinguística. Esta é o resultado do relacionamento entre as diferentes partes do nosso corpo e sua manifestação externa, incluindo a própria voz. Exemplos dessa categoria são: palidez, sudorese, temperatura corporal, tonalidade da voz e qualidade dos sons.

Formalidade. Djajalaksana, Zekavat e Moon (2017) classificaram a formalidade na comunicação em duas categorias. A primeira é a formal. Esta constitui o conjunto de canais e meios de comunicação estabelecido de forma consciente $\mathrm{e}$ deliberada. Ela se refere a canais estruturados e oficialmente aplicados pela gerência. A segunda categoria é a informal. Esta decorre de ambientes de relacionamento e é constituída por conexões pessoais não estruturadas com parceiros selecionados. Ela é utilizada pelos membros da organização para suprir deficiências e minimizar a insegurança do sistema formal de comunicação, aprofundar as agregações do grupo formal e favorecer o conhecimento coletivo.

Localização física. Paasivaara e Lassenius (2001) classificam a comunicação em três categorias. A primeira é a tradicional, composta por projetos realizados por uma empresa em uma única localização física. A segunda é a intrafirma, composta por projetos realizados por uma única empresa e distribuídos em diversas localizações físicas. E por último a interfirma, composta por projetos realizados por diversas empresas distribuídas geograficamente.

Hierarquia. Ao pesquisar sobre as distorções da comunicação em organizações, Larson e King (1996) deram maior foco entre a troca de informações entre supervisores e empregados. Para esses autores, embora reduzir as hierarquias de gerenciamento intermediário ou de nivelamento possa reduzir o nível de ruído dentro do sistema, ele não elimina a distorção, pois os gerentes ainda dependem dos subordinados para obter informações críticas. Isso acaba gerando uma classificação da comunicação, em três categorias, no que diz respeito à hierarquia. A primeira categoria é a vertical, que ocorre entre pessoas em diferentes níveis da hierarquia e pertencentes à mesma unidade de comando. A segunda é a horizontal, que ocorre entre pessoas no mesmo nível da hierarquia e é projetada para melhorar a coordenação do esforço de trabalho. Uma comunicação horizontal eficaz deve impedir a visão de túnel na organização. A terceira é a diagonal, que ocorre entre pessoas em diferentes níveis da hierarquia e pertencentes 
a unidades organizacionais distintas. Ela geralmente é projetada para fornecer informações, coordenação ou assistência a uma ou ambas as partes.

Finalidade. Silveira (2008) categorizou a comunicação em três tipos, conforme a finalidade: coordenação, que visa reduzir o déficit de informações para condução de tarefas e atividades; conhecimento, que visa reduzir a ambiguidade técnica e compartilhar conhecimento, para que membros possam aprender, desenvolver novas habilidades, definir problemas e resolvê-los; e criatividade ou inspiração, que visa aumentar a motivação técnica ou individual.

Método. Para o PMI (2017) há vários métodos de comunicação usados para compartilhar informações entre as partes interessadas de um projeto. Entre eles, três se destacam. O primeiro é o interativo, que ocorre entre duas ou mais partes que estão realizando uma troca de informações multidirecional em tempo real. Este utiliza artefatos de comunicações como reuniões, telefonemas, mensagens instantâneas, algumas formas de mídias sociais e videoconferência. $\mathrm{O}$ segundo é o ativo, na qual a comunicação é encaminhada ou distribuída diretamente para destinatários específicos que precisam receber as informações. Isso garante que as informações sejam distribuídas, mas não que tenham realmente chegado ou tenham sido compreendidas pelo público-alvo. Além disso, os artefatos de comunicação ativa incluem cartas, memorandos, relatórios, emails, faxes, correios de voz, blogs e comunicados à imprensa. Por último, há o método passivo, que é usado para conjuntos de informações grandes e complexos, ou para públicos grandes. Esse método requer que os destinatários acessem conteúdo a seu critério e em conformidade com procedimentos de proteção. Esse método inclui portais da web, sites de intranet e e-learning, e bancos de dados de lições aprendidas ou repositórios de conhecimento.

Abordagem. Para o PMI (2017) diferentes abordagens devem ser aplicadas para atender às necessidades das principais formas de comunicação. As abordagens variam em função do grupo alvo. A primeira delas é a interpessoal, que se refere a informações trocadas entre indivíduos. A segunda relaciona-se com grupos pequenos. Esse tipo de comunicação ocorre dentro de grupos de cerca de três a seis pessoas. A terceira abordagem é a pública. Nesse tipo de comunicação um único palestrante fala para um grupo de pessoas. A quarta abordagem é denominada em massa, pois a comunicação ocorre entre o grupo que envia a mensagem e grupos maiores, às vezes anônimos, para quem as informações são direcionadas. Em geral, não há vínculo entre o grupo emissor da mensagem e os grupos receptores. Por último, destaca-se a abordagem denominada em rede, ou seja, que se refere à comunicação em redes sociais. Esta ocorre de muitos para muitos, e é suportada pela tecnologia e mídia de computação social.

\subsection{Técnicas de Comunicação em Projetos}

Foi utilizada a técnica de revisão sistemática da literatura (RSL) para a identificação e descrição das técnicas de comunicação. A RSL foi baseada nos procedimentos de Kitchenham et al. (2009). O protocolo é constituído pelos itens: questões de pesquisa; base de dados utilizada; critérios e etapas de seleção; e resultados da RSL. 


\subsubsection{Questões da Revisão Sistemática}

Para atender ao primeiro objetivo específico dessa pesquisa, foi formulada a seguinte questão de pesquisa bibliográfica nas bases de dados: quais são as técnicas de comunicação descritas na literatura e aplicadas em projetos de TI?

A seleção contemplou apenas trabalhos científicos considerados relevantes e relacionados com técnicas de comunicação em projetos de TI. O material selecionado foi constituído por teses e textos publicados em conferências ou periódicos. Cabe destacar ainda que a seleção compreendeu apenas trabalhos escritos em língua inglesa, por ser um padrão na área científica e pela relevância dos trabalhos. Além disso, testes realizados nas buscas com a língua portuguesa não retornaram diferenças nos resultados.

\subsubsection{Base de Dados Utilizada}

Os termos de busca foram construídos com base em três palavras-chave relevantes: comunicação (communication); projetos (projects); e técnicas (techniques). Para a palavra-chave 'técnica' foram considerados os sinônimos também: (characteristic, dimension, style, aspect, feature, element, attribute, quality, property, type, model, template, pattern). A estrutura lógica do termo de busca se encontra no apêndice A.

Os termos de pesquisa foram submetidos a cinco bases de dados: $A C M$ Digital Library (https://dl.acm.org/); IEEE Xplore Digital Library (https://ieeexplore.ieee.org/); Science Direct (https://www.sciencedirect.com/); Scopus (https://www.scopus.com/); e Web of Science (https://apps.webofknowledge.com/). Foram identificadas técnicas de comunicação citadas em cada uma das referências.

\subsubsection{Seleção dos Estudos}

Para selecionar somente os trabalhos que atendessem aos objetivos da pesquisa, definiram-se critérios de inclusão e de exclusão. Esta RSL adotou quatro critérios de inclusão:

CI1 O título ou resumo deve abordar características da comunicação em projetos.

CI2 O trabalho deve abordar características da comunicação em projetos.

CI3 O trabalho deve pertencer à área de TI na classificação dos motores de busca.

CI4 O idioma dos trabalhos deve ser o inglês.

Foram adotados critérios de exclusão para trabalhos que:

CE1 Não abordem o tema de comunicação em projetos de TI.

CE2 Atendam apenas a uma ou duas palavras-chave.

CE3 Não foquem nas características da comunicação de forma clara e segregada, e que não permitem extrair informações que respondam as questões de pesquisa.

CE4 Sejam publicados antes de 1999 (amplitude da pesquisa é 2010 a 2019).

CE5 Não permitem acesso, ou seja, não disponíveis integralmente.

CE6 Estejam duplicados na mesma base de dados ou em bases distintas.

Os critérios de qualidade adotados basearam-se na relevância dos artigos. A análise dessa relevância foi realizada em relação à questão da pesquisa bibliográfica. Os artigos foram classificados em três categorias: não abordam técnicas de comunicação; abordam apenas indiretamente essas técnicas; e abordam as técnicas. Os 
artigos classificados nas duas últimas categorias foram selecionados.

A partir da RSL foram extraídos 603 artigos, conforme ilustra a tabela 1. Constam, também, as inclusões e exclusões feitas com base nos critérios de seleção. O trabalho de extração utilizou os softwares StArt e o aplicativo Microsoft ${ }^{\circledR}$ Excel. Ao final foram selecionados 37 artigos, cuja relação se encontra no apêndice B.

Tabela 1 - Quantidade de resultados por motor de busca

\begin{tabular}{|c|c|c|c|c|c|c|c|}
\hline \multirow{2}{*}{\multicolumn{2}{|c|}{ Resultado da extração }} & \multicolumn{5}{|c|}{ Base de Dados } & \multirow{3}{*}{$\begin{array}{r}\text { Total } \\
603\end{array}$} \\
\hline & & ACM & IEEE & S.Direct & Scopus & WoS & \\
\hline Extração inicial & Critérios de inclusão & 8 & 135 & 58 & 338 & 62 & \\
\hline \multirow[t]{2}{*}{ Rejeitados } & Critérios de exclusão & -4 & -126 & -56 & -319 & -56 & -561 \\
\hline & Critérios de qualidade & -1 & -1 & 0 & -2 & -1 & -5 \\
\hline Selecionados & & 3 & 8 & 2 & 17 & 5 & 37 \\
\hline
\end{tabular}

Fonte: elaborada pelo autor

2.2.4 Resultados da RSL sobre as Técnicas de Comunicação em Projetos de TI

$O$ resultado da RSL identificou as técnicas de comunicação em projetos de TI e permitiu agrupá-las em categorias. Essas técnicas representam os meios e canais de comunicação utilizados para troca de informações entre as partes interessadas no projeto. Entre os canais utilizados podem ser citados os orais, os escritos, os eletrônicos e os digitais. Segundo Gillard e Johansen (2004), a decisão da mídia apropriada a ser usada para transmitir a mensagem - telefone, videoconferência, e-mail, conversa, gestos face a face, uma mesa, um gráfico - é de suma importância. Dentro desse contexto, diversos autores analisaram as técnicas de comunicação sob pontos de vista distintos:

Fases do projeto. Bhalerao e Ingle (2010) estudaram as técnicas de comunicação em diversas fases de interação dos projetos, e os resultados mostraram que em todas as fases a comunicação presencial (face a face) entre membros da equipe prevalece; porém, em relação à comunicação com os clientes, tanto e-mails quanto a comunicação presencial são os modos mais efetivos. Outras pesquisas mostraram resultados semelhantes (CHANG; EHRLICH, 2007; JOHANSEN; GILLARD, 2005).
Efetividade da comunicação. Melnik e Maurer (2004) estudaram a efetividade das técnicas em relação ao canal escolhido: quadro de desenhos, telefone, $e$ mail, gravação em vídeo, gravação em áudio, papel. Concluíram que as comunicações presenciais face a face oferecem a perspectiva de uma comunicação mais rica pela capacidade de transmitir várias informações (presença física, inflexão de voz e linguagem corporal).

Sincronicidade. Korkala e Maurer (2014) definiram sincronicidade da mídia como sendo a medida em que uma técnica de comunicação permite que os indivíduos alcancem um padrão compartilhado e coordenado de trabalho. Esses autores classificaram as técnicas de comunicação em projetos pelo seu nível de suporte à sincronicidade. Conferências de vídeo e comunicação face a face têm um nível alto de suporte, teleconferências e chats síncronos possuem um nível médio, enquanto e-mail, mensagens de voz e documentos têm nível baixo. De forma semelhante, Hummel e Rosenkranz (2013) compararam as técnicas de comunicação com suas capacidades de sincronicidade. A eficácia e a eficiência de várias técnicas foram quantificadas usando equações matemáticas. A pesquisa mostrou que a qualidade da comunicação em projetos com urgência foi menor do que em projeto sem urgência. 
As diversas técnicas de comunicação apresentadas na literatura por Chen et al. (2013), Avritzer et al. (2014), Kennedy, McComb e Vozdolska (2011), Bhalerao e Ingle (2010), Melnik e Maurer (2004),
Korkala e Maurer (2014) e Tuomas et al. (2012) foram agrupadas em função de características comuns, usando análise de conteúdo semântica (BARDIN, 2011), e estão apresentadas no quadro 1 .

Quadro 1 -Técnicas de Comunicação

\begin{tabular}{|c|c|}
\hline Técnica & Descrição \\
\hline T01 & $\begin{array}{l}\text { Ferramentas técnicas. Comunicação assíncrona por meio de comentários, textos ou descrições em } \\
\text { códigos-fonte, suítes de desenvolvimento, versionadores, catalogadores ou demais ferramentas técnicas. }\end{array}$ \\
\hline T02 & $\begin{array}{l}\text { Documentos oficiais. Meios oficiais da empresa para compartilhamento e armazenamento de } \\
\text { informações do projeto. Inclui padrões pré-definidos de especificações, relatórios, boletins ou demais } \\
\text { documentos relevantes. }\end{array}$ \\
\hline T03 & $\begin{array}{l}\text { Correio eletrônico. Troca de mensagens por correio eletrônico ou por listas de distribuição - } \\
\text { direcionadas para um ou mais grupos de pessoas. }\end{array}$ \\
\hline T04 & $\begin{array}{l}\text { Conversa presencial. Conversas envolvendo apenas duas pessoas, realizadas obrigatoriamente de forma } \\
\text { presencial (face a face). }\end{array}$ \\
\hline T05 & $\begin{array}{l}\text { Reuniões em grupo. Conversas envolvendo mais de duas pessoas, realizadas obrigatoriamente de forma } \\
\text { presencial (face a face). Assim como as conversas presenciais (T04), podem ter conteúdos variados, de } \\
\text { teor técnico ou funcional, para definições, levantamento de requisitos ou discussões de melhorias. }\end{array}$ \\
\hline T06 & $\begin{array}{l}\text { Telefone ou mensagens de voz. Comunicação verbal síncrona ou assíncrona. A primeira pode ser } \\
\text { realizada por meio de ligações telefônicas, e a segunda ocorre por mensagens de áudio via aplicativos } \\
\text { instantâneos (de celular ou computador) ou via secretária eletrônica. }\end{array}$ \\
\hline T07 & $\begin{array}{l}\text { Mensagens de texto instantâneas. Comunicação escrita e síncrona, realizada por meio de aplicativos de } \\
\text { troca de mensagens de texto instantâneas, programas corporativos ou demais softwares comunicadores. }\end{array}$ \\
\hline T08 & $\begin{array}{l}\text { Conferências. Comunicação verbal, realizada por meio de conferências de áudio (via telefonemas), vídeo } \\
\text { (via sistemas corporativos) ou presenciais (em salas de reunião específicas para este fim). Sempre } \\
\text { envolvendo mais de duas pessoas, na qual todas possuem propriedade de fala. }\end{array}$ \\
\hline T09 & $\begin{array}{l}\text { Apresentaçães. Comunicação verbal direcionada para exibição de temas ou mensagens específicas. } \\
\text { Difere das conferências por existir um ou mais locutores e ouvintes definidos inicialmente. Exemplos são } \\
\text { workshops, plenárias e palestras. }\end{array}$ \\
\hline T10 & $\begin{array}{l}\text { Ambientes colaborativos. Composto por sistemas de troca de informações de forma colaborativa, na } \\
\text { qual os integrantes interagem como contribuidores ou consumidores de informações. Exemplos são } \\
\text { sistemas no formato wiki, fóruns e sistemas com sandbox pré-definidos para contribuição. }\end{array}$ \\
\hline
\end{tabular}

Fonte: elaborado pelo autor

Em complemento a RSL, destaca-se o trabalho de Chaves et al. (2008), que analisou as técnicas de comunicação empregadas na gestão de comunicação em projetos de software, conforme ilustra a figura 1. O trabalho apresentou a importância e a frequência de uso das técnicas categorizandoas em seu nível de formalidade. 
Figura 1 - Importância e frequência de uso das técnicas de comunicação

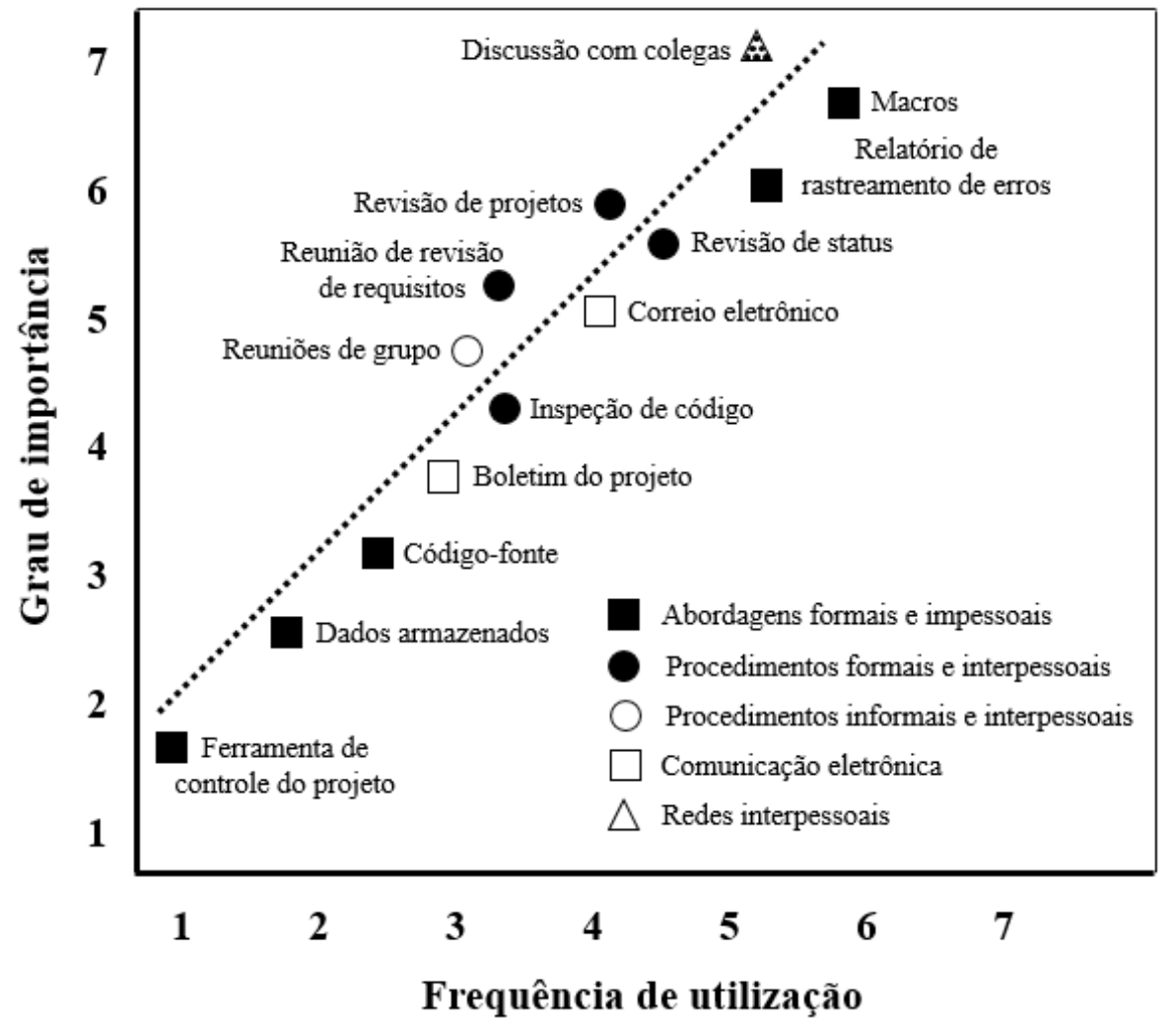

Fonte: adaptado de Chaves et al. (2008).

\section{MÉTODO DE PESQUISA}

Esta pesquisa é fundamentada em uma revisão bibliográfica e em entrevistas com profissionais de gerenciamento de projetos, e apresenta características qualitativas. A seguir serão apresentados o tipo de pesquisa, as fases da pesquisa, bem como os procedimentos de coleta e tratamento dos dados.

\subsection{Características da Pesquisa}

Com base na classificação de pesquisas científicas definidas por Gerhardt e Silveira (2009), esta pesquisa apresenta as seguintes características:

- Natureza. Possui natureza aplicada, pois objetiva gerar conhecimentos para aplicação prática, dirigidos à solução de problemas específicos.
- Tipo. É do tipo descritivo, pois descreve fatos e fenômenos de determinada realidade de maneira exata.

- Estratégia. Trata-se de uma pesquisa ex-post-facto, pois tem como objetivo investigar as técnicas de comunicação em projeto de tecnologia da informação, já finalizados.

- Abordagem. A pesquisa possui abordagem qualitativa e quantitativa (CRESWELL; CLARK, 2013), predominando a abordagem qualitativa. 


\subsection{Fases da Pesquisa}

Esta pesquisa foi desenvolvida em três fases como ilustra a figura 2. A primeira fase compreendeu o levantamento bibliográfico que permitiu identificar as características e as técnicas de comunicação. A segunda fase da pesquisa descreveu os métodos e procedimentos adotados, que estão apresentados nesta seção. Nessa fase foram descritos os instrumentos e os procedimentos de coleta e tratamento de dados, e os sujeitos da pesquisa.

A terceira fase compreende as entrevistas realizadas com os profissionais de projetos de TI, que serviram de base para a confirmação das técnicas de comunicação. A partir delas foram elaboradas a análise dos resultados e as conclusões. Por último, cabe destacar que os dados obtidos na literatura foram coletados no segundo semestre de 2018 .

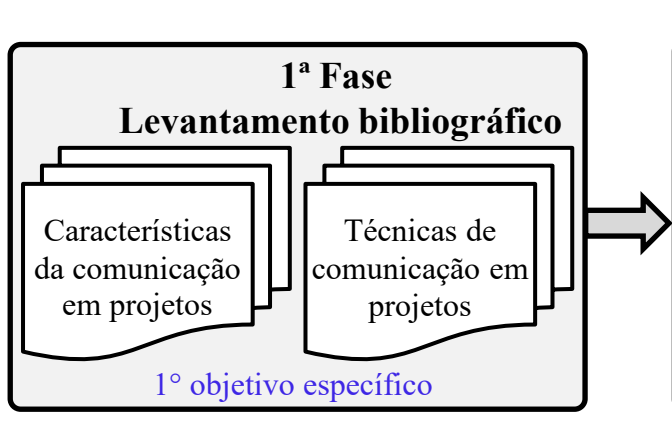

Figura 2 -Fases da pesquisa

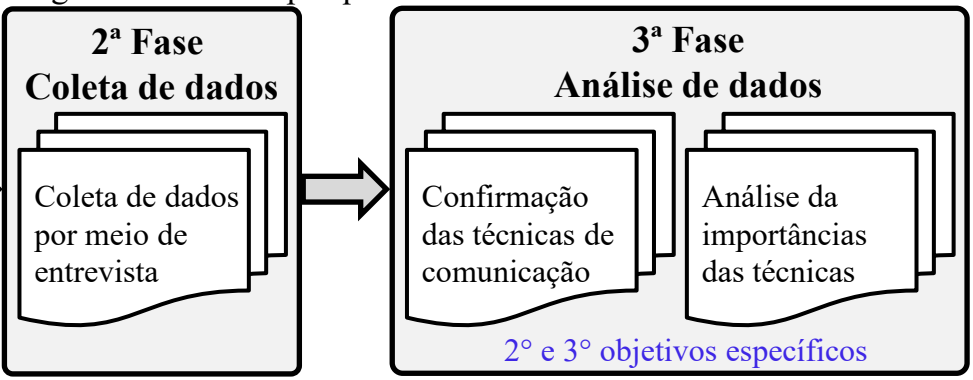

Fonte: elaborada pelo autor

\subsection{Empresa Pesquisada e Sujeitos da Pesquisa}

A empresa selecionada constitui um caso propício para análise em relação ao objetivo desta pesquisa (YIN, 2015). Isso porque, trata-se de uma das maiores e mais importantes instituições financeiras do Brasil, com grande destaque no cenário econômico global e com forte presença nos segmentos de varejo e atacado. Essa empresa gerencia mais de 20 mil colaboradores, tendo atuação em mais de 10 países.

Os investimentos em TI superam dois bilhões de reais por ano, e boa parte desses investimentos é realizado por meio de projetos. Como consequência, essa empresa possui projetos de TI, em todas as áreas da instituição, de porte pequeno ao grande, e com objetivos diversos. Oferece assim, a possibilidade de análise de uma amostra de projeto de TI com características diversas.
Em relação aos sujeitos da pesquisa, adotaram-se os seguintes critérios de seleção: ter experiência em projeto de TI dentro do setor financeiro; atuar em fases distintas dos projetos, desde as discussões para início do projeto até as fases de pós-implantação e rollout; nível mínimo de escolaridade deve contemplar pós-graduação, ou graduação com experiência profissional de no mínimo cinco anos. Além disso, é importante a participação de profissionais de todas as horizontais de atuação, como engenheiros de software, desenvolvedores e líderes de projeto. A amostra mínima adotada foi de dez profissionais de TI que tenham participado de pelo menos um projeto de TI dentro do setor financeiro.

Adotaram-se os seguintes critérios para seleção dos projetos: pertencerem a áreas diversificadas da empresa para considerar contextos distintos; finalizados ou em fase de pós-implantação; e com equipes de tamanho variado, pois o tamanho das equipes pode 
alterar as técnicas de comunicação utilizadas (PMI, 2017).

\subsection{Instrumento, Coleta e Tratamento de Dados}

Dada a natureza aplicada e a abordagem qualitativa predominante da pesquisa, adotou-se a entrevista como instrumento de coleta de dados. As entrevistas foram realizadas com dez profissionais com participação em projetos distintos, relacionados à TI. Em cada entrevista, um participante discursava sobre um único projeto que participou. Após cada entrevista as evidências registradas eram analisadas, e houve convergência delas na décima entrevista.

Adotou-se nesta pesquisa a entrevista semiestruturada. Segundo Manzini (2012), esse tipo de entrevista possibilita a comparação das informações entre os participantes entrevistados. Nelas, o pesquisador pré-estabelece um roteiro, mas permite que o entrevistado fale livremente sobre assuntos que vão surgindo com o desdobramento do tema principal (GERHARDT; ILVEIRA, 2009).

$O$ roteiro da entrevista foi composto por perguntas abertas e fechadas, dividido em três etapas: introdução, na qual são obtidos os dados do entrevistado; contextualização, na qual o projeto é detalhado e compreendido; e proposições da pesquisa, na qual são investigadas as técnicas de comunicação utilizadas.

Dada a característica semiestruturada do instrumento, e em acordo com as recomendações de Manzini (2012), a primeira entrevista das dez foi utilizada como pré-teste para balizar as demais. As entrevistas foram realizadas no primeiro semestre de 2019.

Foi utilizada a técnica de análise de conteúdo semântica no tratamento de dados para categorizar e interpretar os dados coletados nas entrevistas. Segundo Bardin
(2011), a análise de conteúdo semântica permite a exploração qualitativa de mensagens e informações. Além disso, foi adotada uma escala de três pontos para a mensuração da frequência de uso das técnicas de comunicação. Isso porque, segundo Rodriguez (2005), a escala de três pontos é suficiente além de diminuir o tempo na coleta de informação.

\section{APRESENTAÇÃO E DISCUSSÃO DOS RESULTADOS}

Esta seção apresenta o perfil dos entrevistados, a descrição dos projetos analisados nas entrevistas, a confirmação das técnicas de comunicação, e analisa a importância das técnicas. Os resultados são baseados em 10 entrevistas, denominadas E01 a E10.

A primeira entrevista serviu como pré-teste, e por essa razão foi selecionado um entrevistado com experiência em projetos acima da média dos demais entrevistados. A partir do pré-teste foram validados os itens do instrumento. Concluído o pré-teste, o instrumento foi ajustado para sua versão final.

As entrevistas foram realizadas individualmente, em um local reservado, silencioso, sem interferência do meio externo e fora do horário de trabalho. Todos os entrevistados foram informados previamente sobre o objetivo da pesquisa e os temas abordados. As entrevistas foram gravadas com o consentimento dos participantes, para posterior transcrição integral e análise. As transcrições foram realizadas e revisadas manualmente pelo pesquisador, e o tempo médio de cada entrevista foi de 61 minutos, conforme tabela 2 .

\subsection{Perfil dos Entrevistados}

Os entrevistados selecionados atenderam ao perfil previsto. De acordo com a tabela 2, os entrevistados possuem uma média de oito anos de experiência em projeto de TI dentro do setor financeiro. Além disso, 
oito deles possuem pós-graduação, e todos possuem formação relacionada à TI com um mínimo de cinco anos de experiência. Cabe destacar ainda, que no processo de seleção dos entrevistados foi verificado o nível de conhecimento em relação à comunicação em projetos de TI. Somente aqueles que mostraram conhecimento mínimo no tema foram selecionados.

Tabela 2 - Dados dos entrevistados

\begin{tabular}{cccccc}
\hline ID & $\begin{array}{c}\text { Idade } \\
\text { (anos) }\end{array}$ & Escolaridade & Formação & $\begin{array}{c}\text { Experiência } \\
\text { (anos) }\end{array}$ & $\begin{array}{c}\text { Entrevista } \\
\text { (min) }\end{array}$ \\
\hline E01 & 29 & Pós-graduação & SI & 9 & 82 \\
E02 & 25 & Pós-graduação & ENG & 5 & 60 \\
E03 & 26 & Pós-graduação & ENG & 5 & 94 \\
E04 & 28 & Graduação & CC & 7 & 76 \\
E05 & 28 & Pós-graduação & CC & 6 & 47 \\
E06 & 28 & Graduação & ENG & 5 & 60 \\
E07 & 31 & Pós-graduação & ENG & 8 & 54 \\
E08 & 29 & Pós-graduação & SI & 9 & 38 \\
E09 & 32 & Pós-graduação & ENG & 5 & 51 \\
E10 & 36 & Pós-graduação & ENG & 18 & 52 \\
\hline Média & 29 & & & 8 & 61 \\
\hline
\end{tabular}

Fonte: dados da pesquisa

\subsection{Descrição dos Projetos}

Os dez projetos de TI analisados possuem também atividades de desenvolvimento de software em seu escopo, seja de forma parcial ou total. São projetos nos quais os colaboradores atuaram entre os anos de 2016 e 2019. A tabela 3 apresenta os projetos alvo da pesquisa.

As características dos projetos correspondem aos requisitos estabelecidos. Os projetos pertencem a áreas diversificadas: três deles na área de tecnologia, três na área de riscos e os demais em quatro áreas distintas. A grande maioria dos projetos (9 de 10) estavam finalizados ou em fase de pósimplantação, o que permite identificar mais claramente o sucesso obtido no curto ou médio prazo. O tamanho das equipes é variado, apresenta uma média de 49 membros com desvio padrão de 54,4 e tendo a menor equipe com cinco integrantes e a maior com 175. Em relação à abordagem do projeto, destaca-se a divisão quase equitativa dos projetos: sete dos projetos adotaram abordagem ágil e cinco cascata (tradicional).

Tabela 3 - Dados dos projetos

\begin{tabular}{|c|c|c|c|c|c|c|c|c|c|c|c|c|c|c|}
\hline \multirow[t]{2}{*}{ ID } & \multirow[t]{2}{*}{ Área do Projeto } & \multirow{2}{*}{$\begin{array}{l}\text { Nível de } \\
\text { Sucesso }\end{array}$} & \multirow{2}{*}{$\begin{array}{l}\text { Status do } \\
\text { Projeto* }\end{array}$} & \multirow[t]{2}{*}{ Equipe } & \multirow[t]{2}{*}{ Abordagem } & \multicolumn{3}{|c|}{ Funções** } & \multicolumn{6}{|c|}{ Fases que atuou $* * *$} \\
\hline & & & & & & $\mathbf{L}$ & $\mathbf{E}$ & D & 1 & 2 & 3 & 4 & 5 & 6 \\
\hline E01 & Pagamentos & Bom & $\mathrm{F}$ & 175 & Ágil & - & - & & • & • & & - & - & • \\
\hline E02 & Riscos & Bom & $\mathrm{F}$ & 55 & Cascata & & • & - & & - & - & - & • & • \\
\hline E03 & Tecnologia & Bom & $\mathrm{E}$ & 15 & Ágil & • & & & & - & • & - & & \\
\hline E04 & Tecnologia & Regular & $\mathrm{P}$ & 10 & Ágil e Cascata & & & - & & - & - & - & - & - \\
\hline
\end{tabular}




\begin{tabular}{|c|c|c|c|c|c|c|c|c|c|c|c|c|c|c|}
\hline E05 & Riscos & Péssimo & $\mathrm{F}$ & 5 & Ágil & & & - & & $\bullet$ & • & $\bullet$ & - & $\bullet$ \\
\hline E06 & Tecnologia & Regular & $\mathrm{P}$ & 100 & Ágil & $\cdot$ & & & & & - & • & • & • \\
\hline E07 & Unidade Externa & Bom & $\mathrm{F}$ & 10 & Cascata & & • & - & • & $\bullet$ & - & $\bullet$ & $\bullet$ & - \\
\hline E08 & Atendimento & Ótimo & $\mathrm{F}$ & 70 & Cascata & & $\bullet$ & & & $\bullet$ & • & $\bullet$ & $\bullet$ & - \\
\hline E09 & Riscos & Bom & $\mathrm{F}$ & 11 & Ágil & $\bullet$ & & & • & $\bullet$ & - & $\bullet$ & - & - \\
\hline E10 & Rec. Humanos & Bom & $\mathrm{F}$ & 40 & Ágil e Cascata & & $\bullet$ & & • & $\bullet$ & • & & & \\
\hline
\end{tabular}

* E. Em execução. F. Finalizado; P Pós-implantação

** L. líder; E. engenheiro de sistemas; D. desenvolvedor

*** 1. ideação; 2. definição de requisitos; 3. desenvolvimento; 4. teste; 5. implantação e 6. pós-implantação

Fonte: dados da pesquisa

Para completar as características diversas dos projetos, os entrevistados participaram em diversas funções dentro do projeto - líder, engenheiro de sistemas e desenvolvedor - e em pelo menos três fases distintas do projeto. Cabe destacar ainda que, segundo os entrevistados, todos os projetos atingiram um bom nível de sucesso, com exceção dos projetos E05.

\subsection{Confirmação das Técnicas de Comunicação}

No início das entrevistas foi apresentada ao entrevistado a relação de técnicas de comunicação em projetos obtida a partir da RSL. A ideia é que o entrevistado analisasse as técnicas com o intuito de propor alterações, caso fosse necessário. Como consequência, foi recebida uma sugestão para aprimoramento da relação de técnicas com base na realidade da empresa analisada. $\mathrm{O}$ entrevistado E01 destacou a existência de ambientes de reunião em grupo e conversas que possuem itens para a interação entre os participantes. Exemplos são as salas com paredes, mesas de vidro e janelas escrevíveis, painéis digitais e materiais visuais. Essa técnica é de uso recente e pode ser o motivo de não ter relato dela na revisão bibliográfica deste trabalho, mas ela consta de publicações dadas em uma escala ordinal de três pontos: 1 - técnica não utilizada; 2 - técnica pouco utilizada; e 3 - técnica muito utilizada. comerciais voltadas para inovação nas organizações (NEVES, 2018). Assim, foi acrescentada a técnica T11, denominada Ambientes Interativos, para representar essa realidade existente na empresa.

As entrevistas permitiram confirmar essas 11 técnicas de comunicação. Ereaut (2002) descreve algumas sugestões de validade para uma pesquisa qualitativa, entre as quais, cita a Confirmabilidade. Esta referese ao quanto as inferências são suportadas por dados e lógica, livre de preconceitos e julgamentos. Os procedimentos das entrevistas e as inferências realizadas são suportados pelos dados coletados.

\subsection{Técnicas de Comunicação Mais Usadas}

Foi analisada a frequência de uso das técnicas de comunicação. Iniciou-se a pesquisa com cinco entrevistas e uma análise previa dos resultados com base na frequência de uso das técnicas; após sete entrevistas os indicadores foram recalculados; entre a oitava e nona entrevistas, obtiveram-se valores próximos aos existentes; finalmente, após 10 entrevistas notou-se convergência nos resultados, não havendo a necessidade de novas entrevistas. As respostas foram

Os resultados sobre o uso de cada técnica estão apresentados na tabela 4. 
Tabela 4 - Frequência de uso das técnicas de comunicação em projetos

\begin{tabular}{c|ccccc|cccccc}
\hline \multirow{2}{*}{ ID } & \multicolumn{4}{|c|}{ Técnicas mais usadas } & \multicolumn{5}{c}{ Técnicas menos usadas } \\
\cline { 2 - 12 } & T03 & T04 & T07 & T05 & T02 & T01 & T06 & T08 & T10 & T11 & T09 \\
\hline E01 & 3 & 3 & 3 & 3 & 3 & 3 & 3 & 2 & 3 & 3 & 3 \\
E02 & 3 & 3 & 2 & 3 & 3 & 3 & 3 & 3 & 1 & 2 & 1 \\
E03 & 3 & 3 & 2 & 2 & 3 & 1 & 1 & 1 & 2 & 1 & 1 \\
E04 & 3 & 3 & 3 & 2 & 2 & 3 & 2 & 1 & 2 & 2 & 1 \\
E05 & 3 & 2 & 3 & 2 & 1 & 3 & 3 & 1 & 1 & 1 & 1 \\
E06 & 3 & 3 & 3 & 3 & 2 & 2 & 1 & 1 & 3 & 3 & 2 \\
E07 & 3 & 3 & 3 & 2 & 3 & 1 & 3 & 3 & 1 & 3 & 2 \\
E08 & 3 & 3 & 3 & 2 & 3 & 2 & 3 & 2 & 1 & 1 & 1 \\
E09 & 3 & 3 & 2 & 3 & 2 & 3 & 2 & 1 & 1 & 1 & 2 \\
E10 & 3 & 3 & 3 & 3 & 3 & 3 & 2 & 3 & 3 & 1 & 2 \\
\hline Total & 30 & 29 & 27 & 25 & 25 & 24 & 23 & 18 & 18 & 18 & 16 \\
\hline
\end{tabular}

Fonte: dados da pesquisa

A soma das pontuações obtidas pelas técnicas está apresentada em ordem descrente. Por meio do cálculo da mediana foi possível separar as técnicas em dois grupos:

- Mais usadas: correio eletrônico (T03), conversas presenciais (T04), mensagens de texto (T07), reuniões em grupo (T05) e documentos oficiais (T02).

- Menos usadas: ferramentas técnicas (T01), telefone ou mensagens de voz (T06), conferências (T08), ambientes colaborativos (T10), ambientes interativos (T11) e apresentações (T09).

O destaque foi a técnica de correio eletrônico (T03), muito utilizada em todos os projetos, ao contrário da T11, a menos utilizada. Cabe destacar que a técnica T11 é uma das mais recentes e utilizada em situações específicas. Ela foi utilizada majoritariamente em assuntos intrínsecos dos projetos, como em definições técnicas, resolução de problemas e discussões sobre o andamento das atividades. Além disso, a empresa disponibiliza salas para que os colaboradores possam se reunir a qualquer hora para tratar de assuntos de projetos e usar mesas e paredes para escrever e compartilhar suas ideias sobre os projetos.

\subsection{Motivação para o Uso das Técnicas de Comunicação}

Durante as entrevistas foi questionada a motivação para o uso de cada técnica de comunicação nos projetos. As respostas foram agrupadas com base na análise de conteúdo semântica e foram obtidas sete categorias:

- Atividades do projeto: Técnicas usadas em atividades cotidianas do projeto, relacionadas, por exemplo, a listas de pendências, dúvidas, alinhamentos, definição de solução técnica, listas de discussão e resolução de problemas.

- Stakeholder. Técnica de comunicação utilizada com as partes interessadas no projeto: cliente, usuário, executivo e líderes de projetos e departamentos, entre outros. Fazem parte dessa comunicação as especificações de requisitos, boletins de acompanhamento e reports de status.

- Processos. Trata-se da técnica de comunicação relacionada à adoção de metodologias, dos 
processos internos do projeto e da organização, e das cerimônias existentes nas metodologias ágeis. É um tipo de comunicação obrigatória ou extremamente relevante.

- Armazenamento. Refere-se à técnica de comunicação relacionada ao armazenamento de informações que podem ser resgatadas no futuro dos projetos e à formalização de definiçõos ou milestones atingidos no projeto.

- Localização. Trata-se da técnica de comunicação requerida em função da proximidade ou distância física entre equipes.

- Massividade. Técnica de comunicação usada para tratar grande quantidade de mensagens, ligações telefônicas e documentos recebidos. É possível transmitir as mensagens por meio de broadcast. Um exemplo é a comunicação em formato de sala de guerra (warroom), que permite a transmissão de informações em escala para assuntos críticos e momentâneos.

- Informalidade. Técnicas usadas para comunicação informal. Ou seja, usadas entre a equipe do projeto, em grupos de conversas ou em comunicação com líderes.

A tabela 5 apresenta as técnicas utilizadas em função da motivação. A tabela foi dividida em quatro quadrantes em função das medianas relativas às frequências das técnicas e às motivações. Assim, foi possível identificar as técnicas que possuem mais motivações distintas, bem como as motivações que possuem mais técnicas associadas a elas.

Tabela 5 - Motivação para o uso das técnicas de comunicação

\begin{tabular}{|c|c|c|c|c|c|c|c|c|c|c|c|c|}
\hline \multirow[t]{2}{*}{ Motivações } & \multicolumn{11}{|c|}{ Técnicas } & \multirow[t]{2}{*}{ Total } \\
\hline & T03 & T06 & T07 & T09 & T05 & T08 & T01 & T02 & T04 & T10 & T11 & \\
\hline Atividades do projeto & $\bullet$ & $\bullet$ & - & $\bullet$ & - & $\bullet$ & & & $\bullet$ & $\bullet$ & - & 9 \\
\hline Informalidade & • & • & • & & & • & & & • & & & 5 \\
\hline Stakeholder & • & - & & • & & & & • & & & & 4 \\
\hline Localização & & - & • & & - & • & & & & & & 4 \\
\hline Massividade & & & • & • & - & & & & & • & & 4 \\
\hline Processos & & & & $\bullet$ & & & $\bullet$ & $\bullet$ & & & & 3 \\
\hline Armazenamento & - & & & & & & $\bullet$ & & & & & 2 \\
\hline Total & 4 & 4 & 4 & 4 & 3 & 3 & 2 & 2 & 2 & 2 & 1 & 31 \\
\hline
\end{tabular}

Seis técnicas de comunicação ficaram em destaque: correio eletrônico (T03), telefonemas ou mensagens de voz (T06), mensagens instantâneas de texto (T07), apresentações (T09), reuniões em grupo (T05) e conferências (T08). Dessas seis, a maioria (quatro) é baseada em TI. Somente apresentações (T09) e reuniões em grupo (T05) podem utilizar a TI, mas não são necessariamente baseadas nela. Por outro lado, as técnicas com menos motivações de adoção não são baseadas em TI - ferramentas técnicas (T01), documentos oficiais (T02) e conversas presenciais (T04). Isso mostra a relevância da comunicação digital em projetos, ideia corroborada por Chang e Ehrlich (2007) e Herbsleb e Grinter (1999).

\subsection{Pontos Fortes e Fracos das Técnicas de Comunicação}


Os pontos fortes e fracos das técnicas de comunicação foram analisados para as técnicas mais usadas. Pontos fortes são características destacadas pelos entrevistados que contribuem para o sucesso do projeto, e pontos fracos são o contrário. As respostas dos entrevistados foram agrupadas com base na análise de conteúdo semântica e foram obtidas dez categorias, conforme ilustra a tabela 6 .

Tabela 6 - Pontos fortes e fracos das técnicas de comunicação mais usadas

\begin{tabular}{|c|c|c|c|c|c|c|}
\hline \multicolumn{2}{|l|}{ Destaque } & \multicolumn{5}{|c|}{ Técnicas } \\
\hline & & T05 & T04 & T02 & T03 & T07 \\
\hline \multirow[t]{5}{*}{ Pontos fortes } & - Formalização & & & $(+)$ & $(+)$ & \\
\hline & - Agilidade & & & & $(+)$ & \\
\hline & - Alinhamento & $(+)$ & $(+)$ & & & \\
\hline & - Esclarecimento & $(+)$ & $(+)$ & & & \\
\hline & - Suporte à comunicação & & & & & $(+)$ \\
\hline \multirow[t]{5}{*}{ Pontos fracos } & - Não captura falha & & & $(-)$ & $(-)$ & \\
\hline & - Não controla conteúdo & & & & $(-)$ & \\
\hline & - Cobrança excessiva & & $(-)$ & & & \\
\hline & - Excesso de informação & & & & $(-)$ & $(-)$ \\
\hline & - Falta de formalidade & & & & & $(-)$ \\
\hline Classificação & & 2 & 1 & 0 & -1 & -2 \\
\hline
\end{tabular}

Fonte: dados da pesquisa

As técnicas de comunicação com mais pontos fortes foram correio eletrônico (T03),

conversas presenciais (T04) e reuniões em grupo (T05). Por outro lado, a técnica T03 foi a que mais apresentou pontos fracos e a técnica T05 não apresentou nenhum ponto fraco. Cabe destacar também a importância da formalização na comunicação. Ela foi um ponto forte em algumas técnicas e um ponto fraco em outra. A seguir a descrição dos pontos fortes das técnicas de comunicação.

- Formalização. Relacionado à formalidade do trabalho, seja para a divulgação aos stakeholdes ou até mesmo para o registro de requisitos e decisões do projeto.

- Agilidade. Rapidez com que a técnica dissemina informação a ser transmitida, seja de forma individual ou para um grupo de pessoas.
- Alinhamento. Solução para divergências em requisitos e alinhamento de definições dos projetos. É importante para que exista equivalência entre o que é solicitado pelo cliente e o que será entregue pelo projeto.

- Esclarecimento. Tem o poder de esclarecer pontos conflitantes, gerar discussões, evitar falhas e enviar informações sobre $\mathrm{o}$ projeto em massa e de forma clara.

- Suporte à comunicação. Ferramentas desenvolvidas internamente na empresa ou soluções de mercado para suporte à comunicação entre equipes que trabalham em polos distantes.

A seguir a descrição dos pontos fracos das técnicas de comunicação. 
- Não capturam falhas. Falhas em processos internos da empresa não são capturadas por meio dessas técnicas, como erros de solução e redundâncias em procedimentos.

- Não controla conteúdo. Não há capacidade de controle sobre o conteúdo da comunicação por meio da técnica. Por exemplo, o que é combinado versus o que é enviado.

- Cobrança excessiva. Habilitam o acompanhamento do projeto e permitem antecipar datas de entrega. Como consequência, podem derivar para um aumento de pressão sobre a equipe do projeto.

- Excesso de informação. Quando não há restrições a quantidade de dados transmitidos pela técnica, cria uma quantidade excessiva de requisições ou mensagens recebidas pela equipe do projeto.

- $\quad$ Falta de formalidade. Técnicas possibilitam negociações formais por meios informais. Por exemplo, listas de pendências e soluções técnicas controladas com o uso de técnicas informais.

Os resultados obtidos foram comparados com a literatura. A revisão da literatura desta pesquisa caracterizou a comunicação em sete categorias: verbalização, formalidade, localização física, hierarquia, finalidade, método e abordagem. Essas categorias foram referenciadas, direta ou indiretamente, nas análises de motivações, pontos fortes e fracos das técnicas de comunicação. Por exemplo, o baixo nível de formalidade descrito por Djajalaksana, Zekavat e Moon (2017) foi uma das motivações para uso das técnicas de correio eletrônico (T03), conversas presenciais (T04), telefonemas ou mensagens de voz
(T06), mensagens instantâneas de texto (T07) e conferências (T08). Mais ainda, o grau de formalidade apresentou pontos fortes no caso das técnicas de documentos oficiais (T02) e correio eletrônico (T03), e sua ausência despontou como um ponto fraco na técnica de mensagens instantâneas de texto (T07).

Os resultados também foram ao encontro da pesquisa de Chaves et al. (2008). A técnica mais destacada nesta pesquisa foi a reunião em grupo (T05). Esta ficou entre as mais usadas, a que possui mais motivação para adoção e a que tem mais pontos fortes. De forma semelhante, a técnica de reunião em grupo e reunião de revisão de requisitos estão entre as mais importantes na pesquisa de Chaves et al. (2008). Segundo estes autores essas duas técnicas se caracterizam pela interpessoalidade, quer por procedimentos formais ou informais.

\section{CONCLUSÕES}

O objetivo deste trabalho foi analisar as técnicas de comunicação em projeto de TI em uma instituição financeira brasileira. Para atingir esse objetivo, efetuou-se uma pesquisa qualitativa e descritiva com uso da técnica de análise de conteúdo semântica, que contou com o apoio de 10 profissionais com experiência no gerenciamento de projetos em uma instituição financeira brasileira. A resposta à questão de pesquisa e as conclusões deste trabalho estão agrupadas a seguir em função dos objetivos específicos:

- Identificar as principais técnicas de comunicação: foram identificadas dez técnicas de comunicação na literatura, por meio de uma RSL que abrangeu o período de 2010 a 2019.

- Confirmar as técnicas de comunicação: as técnicas foram confirmadas por profissionais com experiência em projeto de TI. A confirmação identificou uma técnica (Ambientes Interativos) não encontrada na RSL, totalizando assim 11 técnicas de comunicação em projetos de TI. 
- Analisar a importância das técnicas: essa análise foi realizada considerando a frequência de uso, as motivações para adoção e os pontos fortes e fracos.

A principal contribuição teórica da pesquisa se refere ao aprimoramento dos frameworks de gerenciamento de projetos. No caso do framework Project Management Body of Knowledge (PMI, 2017), por exemplo, a contribuição ocorre na área de gerenciamento das comunicações. Há a necessidade de maior atenção à comunicação entre os membros da equipe de projeto, em especial às técnicas de comunicação. Nesse caso, destaca-se o uso de técnicas inovadoras baseadas em tecnologia da informação. Isso porque, as técnicas que mais se destacaram foram: correio eletrônico (T03), mensagens instantâneas de texto (T07) e reuniões em grupo (T05). Isso evidencia a evolução no uso de técnicas baseadas em TI.

Essa constatação está em linha com o crescimento da internet e as inserções de TI colaborativa nas organizações e sociedade. $\mathrm{O}$ isolamento social motivado pela pandemia, que se iniciou no começo de 2020, acelerou os processos de transformação digital. Nesse novo cenário, as técnicas de comunicação baseadas em TI tendem a crescer em uso e devem desempenhar um papel de maior relevância nos frameworks de gestão da comunicação em projetos.

A pesquisa também contribuiu com a prática gerencial. Houve ampliação do conhecimento sobre as técnicas de comunicação mais importantes em projetos de TI no contexto brasileiro, que permitem um aumento do nível de desempenho dos projetos de TI. Com base nos resultados das entrevistas foi mapeada uma nova técnica, denominada "ambientes interativos". Esta é composta por meios para a interação entre os participantes, como salas com paredes, mesas de vidro e janelas escrevíveis, painéis digitais e materiais visuais. Essa técnica habilita as características verbal e informal da comunicação em projetos e se alinha a cultura maker, que é baseada em ambientes de colaboração e transmissão de informações entre grupos e pessoas. Além disso, ela se alinha a um novo modelo de gerenciamento, batizado de Gerenciamento 2.0 por McDonald (2011), que reflete o novo paradigma da era da informação.

As contribuições obtidas com esta pesquisa sugerem trabalhos futuros, entre os quais destaca-se a realização de uma pesquisa com características quantitativas, com uso de uma amostra aleatória, que permita verificar as relações sugeridas nesta pesquisa, bem como generalizar o resultado para outras empresas e contextos.

\section{REFERÊNCIAS}

AVRITZER, A. et al. Survivability models for global software engineering. In: IEEE 9th International Conference on Global Software Engineering. p. 100-109, 2014.

BARDIN, L. Análise de Conteúdo. 5. ed. Brasil: Edições 70, 2011, 280p.

BHALERAO, S.; INGLE, M. Analyzing the modes of communication in agile practices.

In: 3rd International Conference on Computer Science and Information Technology. p. 391-395, 2010.

BUCHANAN, J. Measuring up. PM Network, Project Management Institute, v. 22, n. 9, p. 50-55,2008.

CHANG, K.; EHRLICH, K. Out of sight but not out of mind?: Informal networks, communication and media use in global software teams. In: Proceedings of the 2007 conference of the center for advanced studies on Collaborative research. p. 8697, 2007. 
CHAVES, L. E.; NETO, F. H. S.; PECH, G.; CARNEIRO, M. F. S. Gerenciamento da comunicação em projetos. 3 . ed. Rio de Janeiro: FGV Editora, 2008.

CHEN, Q. L. et al. A model for project communication medium evaluation and selection. Concurrent Engineering, v. 21, n. 4, p. 237-251, 2013.

CRESWELL, J. W.; CLARK, V. L. P. Pesquisa de Métodos Mistos: Métodos de Pesquisa. São Paulo: Penso Editora, 2013.

DINSMORE, C.; CAVALIERI, A. Como se Tornar um Profissional em Gerenciamento de Projetos. Project Management Professional. QualityMark, 2003.

DJAJALAKSANA, M. L.; ZEKAVAT, P. R.; MOON, S. Effectiveness of on-site communication in residential housing projects. ISARC. Proceedings of the International Symposium on Automation and Robotics in Construction, 34, p. 1-6, 2017.

EREAUT, G. Analysis and Interpretation in Qualitative Market Research, Sage Publications, London, 2002.

FEBRABAN. Investimentos de bancos com tecnologia aumentam $\mathbf{4 8 \%}$. 2021 . Disponível em: https://febraban.org.br/?idioma=pt-br. Acesso em: 04 maio 2021.

GERHARDT, T. E.; SILVEIRA, D. T. Métodos de Pesquisa. Porto Alegre: UFRGS, 2009.

GILLARD, S.; JOHANSEN, J. Project management communication: a systems approach. Journal of Information Science, v. 30, n. 1, p. 23-29, 2004.
HARTMAN, F.; ASHRAFI, R. Project management in the information systems and information technologies industries. Project Management Journal, v. 33, n. 3, p. 5-15, 2002.

HERBSLEB, J. D.; GRINTER, R. E. Splitting the organization and integrating the code: Conway's law revisited. 21st

International Conference on Software Engineering. ACM, New York, NY, USA, p. 85-95, 1999.

HUMMEL, M.; ROSENKRANZ, C. Measuring the impact of communication in agile development: A research model and pilot test. In: American Conference on Information Systems, 2013.

JOHANSEN, J.; GILLARD, S. Information resources project management communication: personal and environmental barriers. Journal of Information Science, v. 31, n. 2, p. 91-98, 2005.

KENNEDY, D. M.; MCCOMB, S. A.; VOZDOLSKA, R. R. An investigation of project complexity's influence on team communication using Monte Carlo simulation. Journal of Engineering and Technology Management, v. 28, n. 3, p. 109-127, 2011.

KITCHENHAM, B. et al. Systematic literature reviews in software engineering-a systematic literature review. Information and software technology, v. 51, n. 1, p. 7$15,2009$.

KORKALA, M.; MAURER, F. Waste identification as the means for improving communication in globally distributed agile software development. Journal of Systems and Software, n. 95, p. 122-140, 2014.

LARSON, E. W.; KING, J. B. The systemic distortion of information: An ongoing 
challenge to management. Organizational Dynamics, v. 24, n. 3, p. 49-61, 1996.

LU, X.; LIU, L.; LIU, L. Relationship research between communication activities and success indexes in small and medium software projects. In: 1st International Conference on Information Science and Engineering, ICISE 2009, IEEE, p. 50225025, 2009.

MANZINI, E. J. Uso da entrevista em dissertações e teses produzidas em um programa de pós-graduação em educação. Revista Percurso - NEMO, v. 4, n. 2, p. 149-171, 2012.

MCDONALD, P. It's time for management version 2.0: Six forces redefining the future of modern management. Futures, v. 43, n. 8, p. 797-808, 2011.

\section{MEIRELLES, F. S. Estudo dos Gastos e} Investimentos em TI nos Bancos. 2015. Disponível em: https://pesquisaeaesp.fgv.br/institucional. Acesso em: 04 maio 2021.

MELNIK, G.; MAURER, F. Direct verbal communication as a catalyst of agile knowledge sharing. In: Agile Development Conference, p. 21-31, 2004.

NEVES, H. Por que algumas empresas estão implementando laboratórios de inovação maker internos? 2018, outubro. Disponível em: https://www.revistadigital.com.br/2018/10/p or-que-algumas-empresas-estaoimplementando-laboratorios-de-inovacaomaker-internos/. Acesso em: 24 abr. 2019.

PAASIVAARA, M.; LASSENIUS, C. Communication in new product development networks - a case study. In: 8th

International Product Development Management Conference, Enschede Netherlands, p. 711-725, 2001.
PMI. Um Guia do Conhecimento em Gerenciamento de Projetos (Guia PMBOK). 6. ed.: Project Management Institute, 2017.

RODRIGUEZ, M. C. Three options are optimal for multiple-choice items: a metanalysis of 80 years of research.

Educational Measurement: Issues and Practice. v. 24, n. 2, p 3-13, 2005.

RUUSKA, I.; VARTIAINEN, M. Critical project competence: a case study. Journal of Workplace Learning, v. 15, n. 7/8, p. 307312, 2003.

SILVEIRA, F. F. As Práticas de Comunicação em Projetos Globais de Desenvolvimento de Produtos em Empresas Multinacionais Brasileiras. Dissertação (Mestrado) - Universidade de São Paulo, 2008.

TUOMAS, N. et al. Reflecting the choice and usage of communication tools in global software development projects with media synchronicity theory. Journal of Software: Evolution and Process, v. 24, n. 6, p. 677692, 2012.

VASCONCELLOS E; HEMSLEY, J. R. Estruturas das Organizações - estruturas tradicionais, estruturas para inovação, estruturas matriciais. 4. ed. São Paulo: Pioneira, 2002.

VOLLGER, W. Comunicação - fator crítico de sucesso em projetos. [S.1.], 2012. Disponível em:

https://www.tiespecialistas.com.br/comunica cao-fator-critico-de-sucesso-em-projetos/.

Acesso em: 24 abr. 2019.

YIN, R. K. Estudo de Caso: Planejamento e Métodos. 5. ed. Porto Alegre: Bookman, 2015. 


\section{Apêndice A - Termos de busca da RSL}

A seguir é apresentada a estrutura lógica do termo de busca que foi utilizado em cada um dos cinco motores de busca das bases de dados pesquisadas. Os detalhes sintáxicos específicos de cada base de dados foram traduzidos para seu uso, de forma que apenas a estrutura lógica do termo de busca é apesentada abaixo.

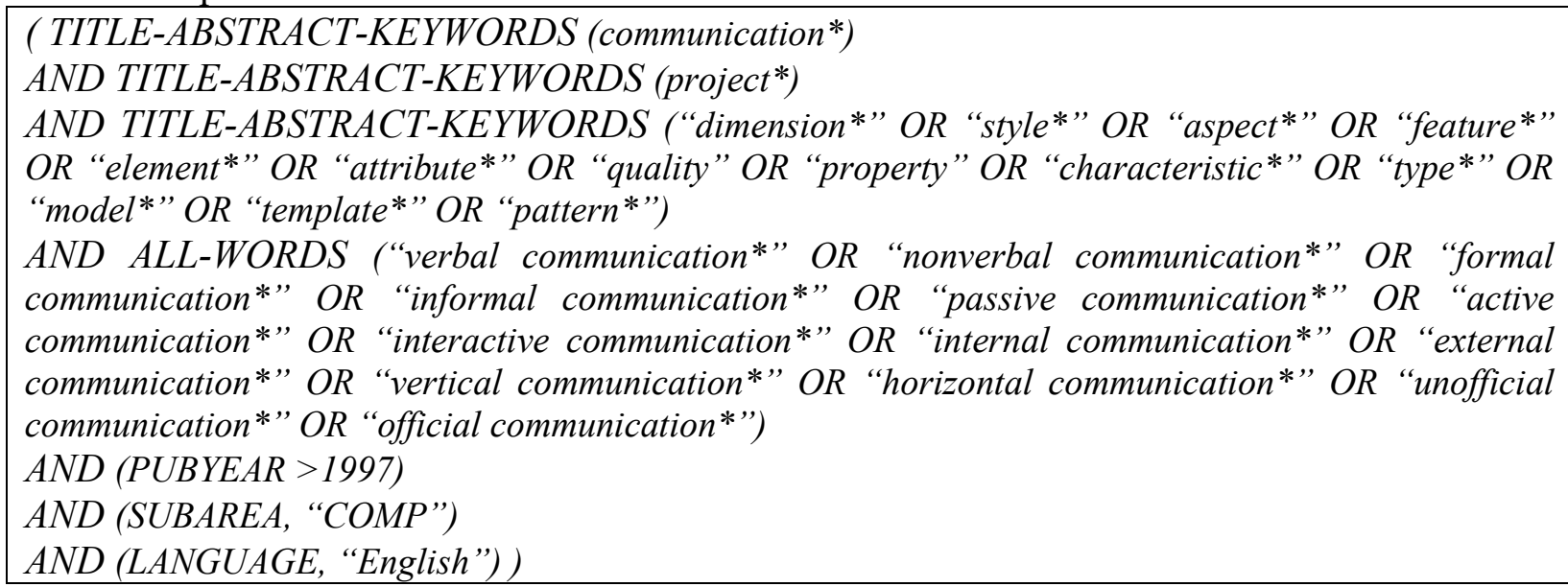

\section{Apêndice B - Artigos Extraídos da RSL}

A relação dos artigos extraídos da RSL está apresentada na tabela 7 em ordem cronológica de publicação.

\begin{tabular}{|c|c|c|}
\hline Título & Autor & Ano \\
\hline Changing interpersonal communication through groupware use & Mark e Wulf & 1999 \\
\hline How to implement a successful communication program: A case study & Carr et al. & 1999 \\
\hline Splitting the organization and integrating the code: Conway's law revisited & Herbsleb e Grinter & 1999 \\
\hline Direct verbal communication as a catalyst of agile knowledge sharing & Melnik e Maurer & 2004 \\
\hline Project management communication: A systems approach & Gillard e Johansen & 2004 \\
\hline $\begin{array}{l}\text { Information Resources Project Management Communication: Personal } \\
\text { and Environmental Barriers }\end{array}$ & Johansen e Gillard & 2005 \\
\hline Communication in Distributed Agile Development: A Case Study & Korkala, Pikkarainen e Conboy & 2007 \\
\hline $\begin{array}{l}\text { Out of sight but not out of mind? Informal networks, communication and } \\
\text { media use in global software teams }\end{array}$ & Chang e Ehrlich & 2007 \\
\hline $\begin{array}{l}\text { Internal and external boundary spanning in outsourced IS development } \\
\text { projects: Opening the black box }\end{array}$ & Vilvovsky & 2009 \\
\hline Analyzing the Modes of Communication in Agile Practices & Bhalerao e Ingle & 2010 \\
\hline $\begin{array}{l}\text { What Information Would You Like to Know about Your Co-worker? A } \\
\text { Case Study }\end{array}$ & Aranda et al. & 2010 \\
\hline $\begin{array}{l}\text { An Investigation of Project Complexity's Influence on Team } \\
\text { Communication Using Monte Carlo Simulation }\end{array}$ & $\begin{array}{l}\text { Kennedy, McComb e } \\
\text { Vozdolska }\end{array}$ & 2011 \\
\hline $\begin{array}{l}\text { How interaction between roles shapes the communication structure in } \\
\text { requirements-driven collaboration }\end{array}$ & Marczak e Damian & 2011 \\
\hline $\begin{array}{l}\text { Projects as Communicating Systems: Creating a Culture of Innovation and } \\
\text { Performance }\end{array}$ & Johannessen e Olsen & 2011 \\
\hline
\end{tabular}




\begin{tabular}{|c|c|c|}
\hline Título & Autor & Ano \\
\hline $\begin{array}{l}\text { Team communications and academic } R \& D \text { performance: } A \text { case of } \\
\text { National Telecommunication Program of Taiwan }\end{array}$ & Hung, Chou e Kuo & 2011 \\
\hline $\begin{array}{l}\text { Elicitation of Communication Inherent Risks in Distributed Software } \\
\text { Development }\end{array}$ & Junior et al. & 2012 \\
\hline $\begin{array}{l}\text { Reflecting the choice and usage of communication tools in global software } \\
\text { development projects with media synchronicity theory }\end{array}$ & Tuomas et al. & 2012 \\
\hline $\begin{array}{l}\text { The impact of communication structure on new product development } \\
\text { outcomes }\end{array}$ & Cataldo e Ehrlich & 2012 \\
\hline A model for project communication medium evaluation and selection & Chen et al. & 2013 \\
\hline $\begin{array}{l}\text { Measuring the impact of communication in agile development: A research } \\
\text { model and pilot test }\end{array}$ & Hummel e Rosenkranz & 2013 \\
\hline $\begin{array}{l}\text { The Role of Communication in Agile Systems Development an Analysis of } \\
\text { the State of the Art }\end{array}$ & Hummel, Rosenkranz e Holten & 2013 \\
\hline Communication: The Foundation of Project Management & Zulch & 2014 \\
\hline Survivability models for global software engineering & Avritzer et al. & 2014 \\
\hline $\begin{array}{l}\text { Waste identification as the means for improving communication in globally } \\
\text { distributed agile software development }\end{array}$ & Korkala e Maurer & 2014 \\
\hline $\begin{array}{l}\text { A conceptual framework to study the role of communication through social } \\
\text { software for coordination in globally-distributed software teams }\end{array}$ & Giuffrida e Dittrich & 2015 \\
\hline Exploring FLOW distance in project communication & Schneider e Liskin & 2015 \\
\hline $\begin{array}{l}\text { The SOCIAL Project Approaching Spontaneous Communication in } \\
\text { Distributed Work Groups }\end{array}$ & Ven et al. & 2015 \\
\hline $\begin{array}{l}\text { Use of social media for internal communication: A case study in a } \\
\text { government organisation }\end{array}$ & Fabre & 2015 \\
\hline $\begin{array}{l}\text { Communication and reputation as essentials for the positioning of } \\
\text { anorganization }\end{array}$ & Karnaukhova e Polyanskaya & 2016 \\
\hline $\begin{array}{l}\text { Managing sub-group interactions and communication in group } \\
\text { collaboration activities }\end{array}$ & Musa, Abidin e Omar & 2016 \\
\hline Project communication management patterns & Muszynska & 2016 \\
\hline $\begin{array}{l}\text { Communication and Coordination Using Facebook: A Case Study of } \\
\text { Distributed Software Development }\end{array}$ & Ferdous e Ikram & 2017 \\
\hline Effectiveness of on-site communication in residential housing projects & Djajalaksana, Zekavat e Moon & 2017 \\
\hline $\begin{array}{l}\text { Exploring horizontal communication of matrix-structured organization } \\
\text { with social penetration theory }\end{array}$ & Lee e Lin & 2017 \\
\hline Patterns of communication management in project teams & Muszynska & 2017 \\
\hline A survey of soft computing applications in global software development & Iftikhar et al. & 2018 \\
\hline $\begin{array}{l}\text { Analysis of the online interactions of students in the project management } \\
\text { learning process }\end{array}$ & Olarte-Valentín et al. & 2018 \\
\hline
\end{tabular}

${ }^{i}$ Graduação em Sistemas de Informação pela Universidade de São Paulo (2010) e MBA em Finanças pela Fundação Getúlio Vargas (2015).

ii Graduado em Engenharia Mecânica (EPUSP) pela Universidade de São Paulo (1985). Possui mestrado (2000) e doutorado (2005) em Administração pela Universidade de São Paulo. 